\title{
Will ORBITA change my practice? ORBITA trial: Objective Randomised Blinded Investigation with optimal medical Therapy of Angioplasty in stable angina
}

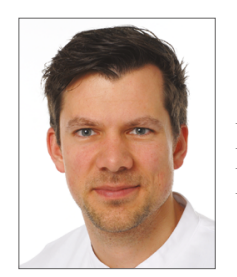

Robert Schueler ${ }^{1 *}, \mathrm{MD}$; Rasha Al-Lamee ${ }^{2}, \mathrm{MD}$; Felix Mahfoud ${ }^{3}, \mathrm{MD}$;

Davide Capodanno ${ }^{4}, \mathrm{MD}, \mathrm{PhD}$; Mirvat Al Asnag5, MD; Michael Haude 6 , MD

1. Department of Cardiology and Angiology, Elisabeth Hospital, Essen, Germany; 2. National Heart and Lung Institute, Imperial College London, London, and Imperial College Healthcare NHS Trust, London, United Kingdom; 3. Department of Internal Medicine III, Cardiology, Angiology and Intensive Care Medicine, Saarland University, Homburg, Germany; 4. Division of Cardiology, CAST, P.O. "Rodolico", Azienda Ospedaliero-Universitaria "Policlinico-Vittorio Emanuele”, University of Catania, Catania, Italy; 5. Cardiac Center, King Fahd Armed Forces Hospital, Jeddah, Saudi Arabia; 6. Städtische Kliniken Neuss, Lukaskrankenhaus GmbH, Neuss, Germany

\section{Introduction to the session}

This summary report aims to capture the content of the session at EuroPCR 2018 that reviewed the implications for clinical practice of the ORBITA trial, in order to share a critical analysis of the trial and report the views expressed in the interactive discussion. This article does not constitute an independent review of the topic by the authors.

\section{Impact of ORBITA}

The impact of percutaneous coronary interventions (PCI) for stable angina on symptoms and quality of life compared to medical treatment alone is not fully understood ${ }^{1,2}$. The Objective Randomised Blinded Investigation with optimal medical Therapy of Angioplasty in stable angina (ORBITA) trial is the first randomised trial to investigate the influence of PCI in a sham-controlled fashion on angina symptoms and exercise time ${ }^{3}$. The results of the ORBITA trial showed that, in patients with stable angina, PCI does not result in greater improvements in exercise times or chest pain frequency compared with a sham procedure and medical treatment, although all included patients had anatomically and/or functionally significant stenosis. The design and results of ORBITA were discussed at a EuroPCR 2018 "Will this trial change my practice?" session. An initial audience poll showed that more than $50 \%$ of attendees felt that ORBITA had not relevantly changed their clinical practice regarding PCI for stable angina patients.

\section{Do we really need a change in clinical practice?}

While summarising the study results, the role of modern communication media was recognised. Social networks (for example, Twitter with more than 800 "\#ORBITA" on the first day after publication of the trial results) have played a major role in disseminating and interpreting the study findings.

ORBITA was primarily designed to test the effect of PCI on angina and not on ischaemia reduction. Apparently, there was a misconception within the cardiology community: ORBITA did not show negative results for PCI in stable angina. First and foremost, the trial demonstrated the placebo effects that invasive

*Corresponding author: Department of Cardiology and Angiology, Elisabeth Hospital, Klara-Kopp-Weg 1, 45138 Essen, Germany.E-mail:R.Schueler@contilia.de 
procedures might have on patients' symptoms and quality of life. Of note, despite ORBITA being described as the "last nail in the coffin of PCI for stable angina"4, it showed that PCI with drug-eluting stent (DES) implantation was superior to a sham PCI in reducing ischaemia in stable angina patients and that $85 \%$ of sham patients chose to undergo PCI at the study end after unblinding.

ORBITA should not lead to a definite change in PCI practice for stable angina. It should, however, possibly change our views on patients' symptoms and treatment alternatives to PCI.

\section{Case presentation}

The case of a 60 -year-old male, very active patient with reproducible chest pain was discussed. During exercise test he had ST depression in leads V4-6 and stress echocardiography demonstrated hypokinetic apical anterior and anteroseptal myocardial segments. Angiography showed a severe proximal LAD stenosis, and FFR and iFR measurements of the LAD stenosis were highly pathologic. The patient was then randomised and up-titrated to $10 \mathrm{mg}$ of bisoprolol and $5 \mathrm{mg}$ of amlodipine per day. Thereafter, he presented with an angina frequency of 100 in the Seattle Angina Questionnaire and was able to attend his sports classes again without angina. The discussion that followed highlighted the importance of putting into perspective the individual conception of symptoms and situation of life of the patients undergoing PCI for stable angina.

However, in an audience poll after the case presentation, almost $100 \%$ of the attendees opted for stenting in this particular case, recognising that clinical reality in a patient with angina and positive non-invasive testing would be stenting without additional functional measurements.

Additional questions were raised about study endpoints and whether the sham design of the study could be perceived as "ethically questionable" by some.

\section{What was known from sham-controlled trials before ORBITA?}

As just stated, one of the major points of critique was the study's sham design. Out of 24 sham-controlled studies, 16 failed to reach pre-specified endpoints and, as a result, a number of procedures, in which negative sham-controlled results were reported, have been abandoned. Sham-controlled research is not well accepted in the clinical cardiology community, as individual patients allocated to sham procedures are exposed only to risk without potential for benefit, in contrast to placebo-controlled trials with pharmaceuticals. Of note, in the ORBITA trial the number of serious adverse events in the control group was higher compared to the PCI group (8/95 vs. 0/105), and 4 of 95 patients in the control group required stenting due to coronary dissection during the sham procedure ${ }^{3}$. As both patients and physicians might be reluctant to participate in a sham-controlled trial, thus promoting a certain selection bias, sham might not be a universal solution for internal and external validatation of a new product or procedure. However, sham-controlled studies might contribute to stop development of interventions or procedures which are ineffective.

The ORBITA trial highlights the value of sham controls to balance out a certain procedural placebo effect, comparably noted, for instance, in the SYMPLICITY HTN-3 trial ${ }^{5}$.

The specific design of a sham-controlled study might assist in ruling out "non-device" effects and might therefore be useful to establish "proof of concept" level of efficacy evidence for new device-based therapies.

\section{Review of the ORBITA study design}

The ORBITA trial was a multicentre, randomised trial that was carried out at five study sites in the United Kingdom and included 200 patients, of whom 105 were in the PCI group and 95 in the sham group. Eligible patients had angina or equivalent symptoms and at least one angiographically significant lesion $(\geq 70 \%)$ in a single vessel that was suitable for PCI. Exclusion criteria were acute coronary syndrome, left main stem, multivessel disease and other conditions that might have obscured symptoms and study results ${ }^{3}$. Eligible patients were selected after diagnostic angiography. The primary endpoint was the difference in exercise time increment between the groups.

The trial was extremely well designed, with thorough evaluation of myocardial ischaemia pre and post procedure. The study comprised two consecutive phases with a six-week medical optimisation period, during which initiation and up-titration of antianginal therapy was achieved. Patients then had baseline pre-randomisation examinations, including stress echocardiography, followed by the randomised blinded procedure. Patients underwent thorough follow-up evaluation six weeks after inclusion. At enrolment, patients had to complete different questionnaires (Seattle Angina Questionnaire, EuroQol 5 dimensions). During the first six weeks, patients had telephone calls with a consulting cardiologist one to three times per week. All patients were followed up for six weeks with re-evaluation of quality of life and angina frequency and intensity. Patients in ORBITA received somewhat more medical support than patients in "normal life" would have, which might have further promoted a placebo effect. One further point of critique was that ORBITA might not reflect typical patients undergoing PCI, since only single-vessel disease and relatively fit patients were included, which might have introduced a selection bias.

However, ORBITA failed to reach its pre-specified primary endpoint: the increase in exercise time was 28.4 seconds in the 104 patients with available follow-up data in the PCI group (95\% CI: 11.6-45.1) and 11.8 seconds in the 90 patients with available follow-up data in the sham group (95\% CI: -7.8-31.3) (Figure 1).

Although PCI did not increase exercise time in the ORBITA trial, secondary outcomes, such as freedom from angina (PCI group $49.5 \%$, sham group $31.5 \%, \mathrm{p}=0.006$ ) or peak stress wall motion index scores (PCI group -0.08, sham group 0.02, $\mathrm{p}=0.0011$ ), were positive for PCI treatment. Although the trial did not meet its primary endpoint, interpretation and judgement of the ORBITA results were mainly based on personal preconceptions. Despite 


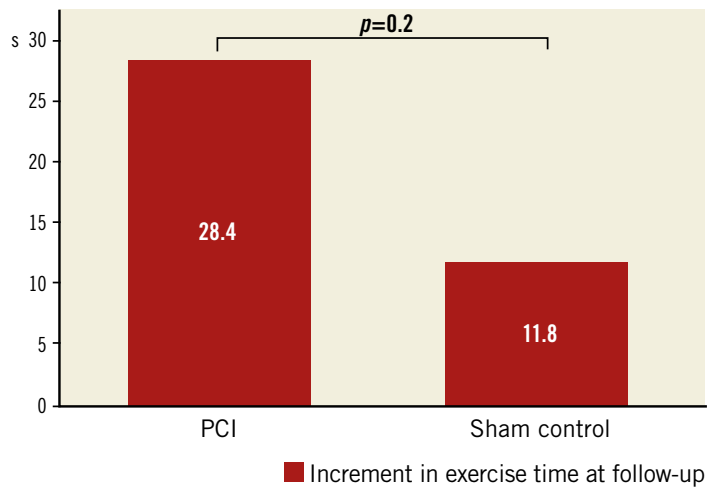

Figure 1. Primary endpoint increment of exercise time prerandomisation to follow-up after six weeks.

being powered for an exercise-based "soft" endpoint, the patient numbers might have been too small to demonstrate that PCI does not result in greater improvements in exercise times or angina frequency compared with a sham procedure, given the rather short follow-up period of six weeks.

\section{Case resolution}

Before unblinding, the patient again underwent stress echocardiography that revealed persisting hypokinesia in the myocardial segments supplied by the stenotic LAD. Unblinding showed that the patient had been randomised to the sham group. Although free of angina, he opted for PCI after exiting the study because of fatigue under maximal antianginal therapy. This exemplary case from the trial illustrates that not only should functional or angiographic evaluation trigger PCI in stable angina patients, but that there should also be thorough consideration of the patients' subjective symptoms and living conditions.

\section{Closing remarks}

Before ORBITA, PCI versus medical therapy alone in patients with stable angina had shown only modest benefit in outcomes or myocardial infarction incidence. Furthermore, in contrast to ORBITA, most trials on PCI in stable angina patients had used outdated drug-eluting stent platforms; results might therefore not apply to current practice. Despite having missed the primary endpoint, ORBITA demonstrated that PCI improves freedom from angina with a very favourable number needed to treat (NNT) of 5. The trial proved that functional testing with FFR/iFR showed a linear association with the degree and the extent of ischaemia, and with ischaemia improvement following $\mathrm{PCI}^{7}$. The results of ORBITA apply to patients with stable anginal symptoms and single-vessel disease who are suitable for PCI and not to patients with acute coronary sydrome, left main or multivessel disease. However, the relationship between angiographic coronary stenosis and ischaemic relief is apparently more complex than thought. Although controversially discussed, ORBITA might have the potential to change clinical practice, since it readjusts interventionalists' focus on patient preference, having demonstrated that antianginal medication alone might be a reasonable option in stable angina patients. ORBITA also showed that PCI improves ischaemia and has given a strong signal that PCI might lead to more freedom from angina compared to medical therapy alone (Table 1 ).

\section{Table 1. Will ORBITA change my practice?}

\section{Major arguments for a change in practice}

- In patients with stable angina, $\mathrm{PCl}$ did not result in greater improvements in exercise times or chest pain frequency compared with a sham procedure and medical treatment.

- $\mathrm{PCl}$ did not result in improvement of quality of life.

\section{Major arguments against a change in practice}

ORBITA demonstrated that $\mathrm{PCl}$ improves freedom from angina with a number needed to treat (NNT) of 5 .

- ORBITA applies to patients with stable anginal symptoms and single-vessel disease and not to patients with acute coronary syndrome, left main or multivessel disease and might not reflect clinical reality.

A possible ORBITA II trial should comprise longer followup time and should include patients with multivessel disease. Furthermore, exercise time might not have been the most meaningful primary endpoint. The findings of ORBITA should be validated in a larger, randomised controlled trial.

\section{Final audience poll}

In a final audience poll, most of the attendees voted that ORBITA might impact on their clinical practice, with regard to understanding patients' subjective symptoms since the trial results suggest that symptomatic improvement from PCI in stable angina contains a certain placebo component. However, ORBITA might not change practice decisions or numbers of PCI procedures in symptomatic patients with stable angina who are good candidates for revascularisation.

\section{Conflict of interest statement}

The authors have no conflicts of interest to declare.

\section{References}

1. Boden WE, O'Rourke RA, Teo KK, Hartigan PM, Maron DJ, Kostuk WJ, Knudtson M, Dada M, Casperson P, Harris CL, Chaitman BR, Shaw L, Gosselin G, Nawaz S, Title LM, Gau G, Blaustein AS, Booth DC, Bates ER, Spertus JA, Berman DS, Mancini GB, Weintraub WS; COURAGE Trial Research Group. Optimal medical therapy with or without PCI for stable coronary disease. N Engl J Med. 2007;356:1503-16.

2. Weintraub WS, Spertus JA, Kolm P, Maron DJ, Zhang Z, Jurkovitz C, Zhang W, Hartigan PM, Lewis C, Veledar E, Bowen J, Dunbar SB, Deaton C, Kaufman S, O'Rourke RA, Goeree R, Barnett PG, Teo KK, Boden WE; COURAGE Trial Research Group, Mancini GB. Effect of PCI on quality of life in patients with stable coronary disease. $N$ Engl J Med. 2008;359:677-87. 
3. Al-Lamee R, Thompson D, Dehbi HM, Sen S, Tang K, Davies J, Keeble T, Mielewczik M, Kaprielian R, Malik IS, Nijjer SS, Petraco R, Cook C, Ahmad Y, Howard J, Baker C, Sharp A, Gerber R, Talwar S, Assomull R, Mayet J, Wensel R, Collier D, Shun-Shin M, Thom SA, Davies JE, Francis DP; ORBITA investigators. Percutaneous coronary intervention in stable angina (ORBITA): a double-blind, randomised controlled trial. Lancet. 2018;391:31-40.

4. Brown DL, Redberg RF. Last nail in the coffin for PCI in stable angina? Lancet. 2018;391:3-4.

5. Bhatt DL, Kandzari DE, O’Neill WW, D'Agostino R, Flack JM, Katzen BT, Leon MB, Liu M, Mauri L, Negoita M, Cohen SA, Oparil S, Rocha-Singh K, Townsend RR, Bakris GL; SYMPLICITY HTN-3 Investigators. A controlled trial of renal denervation for resistant hypertension. $N$ Engl J Med. 2014;370: 1393-401.
6. Byrne RA, Capodanno D, Mahfoud F, Fajadet J, Windecker S, Jüni P, Baumbach A, Wijns W, Haude M. Evaluating the importance of sham controlled trials in the investigation of medical devices in interventional cardiology. EuroIntervention. 2018; 14:708-15.

7. Al-Lamee R, Howard JP, Shun-Shin MJ, Thompson D, Dehbi HM, Sen S, Nijjer S, Petraco R, Davies J, Keeble T, Tang K, Malik IS, Cook C, Ahmad Y, Sharp ASP, Gerber R, Baker C, Kaprielian R, Talwar S, Assomull R, Cole G, Keenan NG, Kanaganayagam G, Sehmi J, Wensel R, Harrell FE, Mayet J, Thom SA, Davies JE, Francis DP. Fractional Flow Reserve and Instantaneous Wave-Free Ratio as Predictors of the PlaceboControlled Response to Percutaneous Coronary Intervention in Stable Single-Vessel Coronary Artery Disease: PhysiologyStratified Analysis of ORBITA. Circulation. 2018 May 22. [Epub ahead of print]. 